\title{
Vers une éthique du futur institutionnelle. Les concepts de responsabilité dans la RSE à la lumière del'héritagede Jonas
}

\author{
Towards an institutional ethics of the future. The \\ concepts of responsibility in Corporate Social \\ Responsibility in the light of Jonas's Heritage
}

EMMANuel PICAVET (iDa

\section{Résumé ${ }^{1,2}$}

Cette contribution est consacrée à la signification actuelle et aux défis institutionnels de I"éthique du futur" au sens de Jonas. On examine d'abord, autour d'enjeux qui sont actuellement au cœur des démarches de Responsabilité sociale des entreprises, les dimensions collective, institutionnelle et partenariale de la responsabilité. Dans un second temps, c'est la préservation des conditions de l'action qui est l'objet de l'attention, dans la continuité de préoccupations de Hans Jonas et en lien avec une perspective collective sur l'action individuelle. Ces développements conduisent à des conclusions au sujet des idées courantes sur le partage des responsabilités dont serait porteuse la Responsabilité sociale des entreprises.

\footnotetext{
a Directeur du Centre de Philosophie Contemporaine de la Universitè Paris 1 Panthéon-Sorbonne, Paris, France. Doctorat en Philosophie, e-mail: emmanuel.picavet@univ-paris1.fr

${ }^{1}$ Ce texte provient de la conférence donnée à l'université pontificale de Sangiago du Chili dans le colloque organisé à l'occasion des "40 ans du Principe responsabilité" d'Hans Jonas, les 2 et 3 octobre 2019. L'auteur remercie sincèrement les organisateurs, Eric Pommier et Luca Valera, pour leur accueil très généreux et pour les utiles discussions transcontinentales qui ont pris corps à l'occasion de cet événement.II remercie aussi Gabriel Vidal Quinones pour la traduction espagnole très attentive de la version initiale de la conférence, qui a fourni l'occasion de clarifier certains points.

${ }^{2}$ Ce travail s'inscrit plus particulièrement dans l'axe RSE de I'ISJPS et dans le projet Chronos de la Chaire Ethique et Finance (FMSH-CEM). Je remercie Christian Walter et Kathia Martin-Chenut pour de nombreux échanges fructueux à propos des questions théoriques et pratiques de la responsabilité partagée.
} 
Mots-clé: Éthique. Institution. Jonas (Hans). Responsabilité Sociale des Entreprises. Responsabilité.

\section{Abstract}

This contribution is devoted to the current meaning and the institutional challenges of the "ethics of the future" in the sense of Jonas. We first examine, about issues that are currently at the heart of Corporate Social Responsibility, the collective, institutional and stakeholderrelated dimensions of responsability. The preservation of the conditions of action will next be under focus, in the continuity of Hans Jonas' concerns, in the framework of a collective perspective on individual action. These developments lead to conclusions about common ideas about the sharing of responsibilities which Corporate Social Responsibility exemplifies.

Keywords: Ethics. Corporate Social Responsibility. Institutions. Jonas (Hans). responsibility.

\section{Introduction}

On a retenu du Principe responsabilité une leçon capitale: la compréhension de la coopération sociale, de l'activité économique et du rapport à la nature ne peut continuer à se développer sans prendre en compte les effets agrégés des actions. C’est le cas, en particulier, quand les risques pour le cadre de l'activité humaine, et pour la vie humaine elle-même, sont très grands. Plus que d'autres philosophes du XXe siècle, Jonas a attiré l'attention, spécialement dans Le Principe responsabilité, sur notre environnement de vie et il est bien naturel qu'il soit aujourd'hui sollicité par les philosophies de l'environnement et de la nature. A ce titre également, son œuvre est une référence pour le mouvement et les initiatives qui se réclament de la « Responsabilité Sociale des Entreprises » (RSE) et, plus largement, pour les initiatives institutionnelles et entrepreneuriales qui concernent la prise de responsabilitésociétale et environnementale. Elle constitue une source d'inspiration, très généralement, pour les travaux consacrés à la responsabilité sociale des organisations (quelle que soit leur nature).

Le pessimisme s'est nourri de la pensée de Jonas pour des raisons qui tiennent au sérieux avec lequel Jonas a pris en compte les résultats agrégés de l'inaction, de l'entraînement et du fatalisme. Face aux défis de la technologie, du contrôle social et de 
l'environnement ${ }^{3}$, ces résultats peuvent, de fait, être extrêmement préoccupants. L'émergence contemporain de la notion de « risque climatique », englobant des effets très larges de l'interaction des activités humaines et du système de la nature, vient rappeler que les mauvaises conséquences du défaut d'adaptation aux risques peuvent concerner les conditions les plus générales et les plus fondamentales de la vie humaine. Si cette dimension « conséquentialiste » de la pensée de Jonas (selon la terminologie souvent adoptée en philosophie) a nourri un certain radicalisme dans le pessimisme, recouvrant notamment le genre de pessimisme de principe à propos de la technique qui a été étudié avec soin et dénoncé en philosophie ${ }^{4}$, ne peut-elle aussi nourrir le progressisme d'une certaine manière? Le Principe responsabilité (par exemple au chap. V) est aussi un livre fondamental sur le progrès, après tout.

Malgré ses limites et en dépit des déceptions qu'il engendre, il se peut que le mouvement de la Responsabilité Sociale des Entreprises (parmi d'autres tendances de la pensée et de l'action dans le monde contemporain) en porte témoignage, du fait de son insistance sur l'enregistrement des progrès liés à une coopération organisée qui, dans les grandes lignes, rencontre des aspirations dont Hans Jonas a montré l'importance. Sur la base des préoccupations qui s'expriment dans son œuvre, la réflexion sur la prise de responsabilité s'est orientée vers un niveau spécifique de rationalité et de : celui de la coopération sociale qui prend une forme institutionnelle. Or, ce niveau présente une importance toute particulière pour la RSE.

Est-ce à dire que la RSE soit, pour autant, à même de prendre en charge dans son intégralité l'appel à la responsabilité que nous associons spontanément à l'héritage intellectuel et moral de Jonas? Ses limites doivent parfois être soulignées, et l'on ne s'en fait pas faute dans un contexte contemporain marqué par une défiance souvent très grande à l'endroit des institutions et des organisations. Les limites ressortiront d'autant plus clairement que l'on voudra bien se consacrer, un moment, à prendre au sérieux également ce qui relève d'un apport positif. On se proposera ici d'en examiner quelques aspects, spécialement ceux qui concernent la recherche de normes et de

\footnotetext{
${ }^{3}$ Hans Jonas, Le principe responsabilité (tr. fr. Jean Greisch de Das Prinzip Verantwortung, Francfort, Insel Verlag, 1979). Paris : Les Editions du Cerf, 1990.

${ }^{4}$ V. en particulier: Jean-Pierre Séris, La Technique, Paris, Presses Universitaires de France, 1994. Pour un autre point de vue, tout différent, v. Jean-Pierre Dupuy, Pour un catastrophisme éclairé, Paris, Seuil, 2002.
} 
cadres d'action adaptés. Dans un second temps, on se tournera vers la dimension collective et institutionnelle du souci - lui aussi jonassien - de la préservation des cadres de l'action proprement humaine.

\section{Spécificités du volet institutionnel de la responsabilité collective face au futur: comment prendre en compte la vie institutionnelle comme telle?}

\section{De l'"éthique du futur" à la RSE: une éthique de la coopération}

Pour tenir compte adéquatement des risques auxquels nous faisons face, il est indispensable, selon Jonas, de valoriser socialement les capacités collectives d'innovation. Cela s'inscrit pleinement dans les perspectives dessinées par l'auteur pour une « heuristique de la peur » mais aussi pour une " éthique du futur », qui est fondamentalement une éthique qui prépare au futur et qui transforme le futur en problème philosophique, plutôt qu'une éthique située dans l'avenir ou une éthique qui s'adapterait à des conjectures sur l'avenir (c'est là une source de malentendus comme on le sait). Pour Jonas, en effet, tenir compte de l'avenir ne peut se limiter à agiter des craintes ou à flatter des espoirs infondés : nous avons des capacités susceptibles d'être valorisées positivement. D'une certaine manière, au plan collectif, l' «éthique du futur» de Jonas est l'une de ces approches de la «mise en capacité» qui sont volontiers mises en relief, aujourd'hui, dans les discours sur l'autonomie. Les capacités humaines pertinentes concernent la technologie mais aussi la société, les modes de vie, les formes de travail. Le travail intellectuel n'en est pas exclu, bien au contraire, et pas davantage les relations entre le travail intellectuel et les institutions ${ }^{5}$.

C'est ainsi, sur une base très large, la coopération qui est à penser, dans un grand effort philosophique pour donner au futur sa place dans le présent. A cet égard, rien n’est plus éloigné des idées de Jonas qu'une perspective purement gestionnaire (en un sens trop étroit) sur la correction des effets négatifs des

\footnotetext{
${ }^{5}$ Outre Le Principe responsabilité, v. notamment à ce propos: Pour une éthique du futur (tr. par S. Cornille et Ph. Ivernel de Philosophie, Rückschau und Vorschau am Ende des Jahrhunderts [Frankfurt : Suhrkamp 1993] et partiellement de Philosophische Untersuchungen und metaphysische Vermutungen [Frankfurt am Main \& Leipzig: Insel Verlag, 1992]. Paris: Payot et Rivages, 1998.
} 
interactions humaine, qui s'appuierait seulement sur des intérêts préexistants et des formes de comportement prévisibles sur la base de ces intérêts. A bonne distance de ce modèle comportementaliste aujourd'hui prédominant dans les approches relevant de l'organisation paternaliste et «incitative » des mécanismes sociaux, il s'agit de prendre en compte et de mobiliser l'aptitude des hommes à la coopération, au travail commun qui affecte tous ceux qui y participent. Il est clair que ce travail commun est de nature à faire évoluer leurs conceptions et leurs attitudes.

Ce que l'on doit aussi retenir à coup sûr de la pensée de Jonas dans les réflexions sur les responsabilités face au futur, c'est la recherche, dans et pour la responsabilité, de fondements de l'action collective. La responsabilité dont il s'agit est orientée vers la préservation adéquate de la figure humaine, de l'agir humain dans sa dignité, et même de la philosophie comme telle. Cette responsabilité est individuelle mais aussi collective et, plus précisément, institutionnelle. C'est notamment au plan institutionnel que nous pouvons nous porter garants de l'existence dans des conditions convenables et de l'expression proprement humaine d'autrui. Cela peut aussi se laisser décrire en termes de préservation de l'humanité dans des conditions dignes d'existence et d'aptitude aux choix.

La double dimension de la responsabilité et de l'institutionnalisation est capitale pour faire fructifier l'apport durable des recherches philosophiques de Jonas. Elle se retrouve aujourd'hui avec une certaine clarté dans le mouvement de la Responsabilité Sociale des Entreprises. On y décèle aussi le souci de l'action convenablement préparée : initiatives de l'entrepreneur, initiatives des collaborateurs (salariés) de l'entreprise, élaboration de la stratégie d'entreprise, initiatives dialogiques et pragmatiques des «parties prenantes » avec lesquelles l'entreprise travaille (par exemple les fournisseurs, les consommateurs, les organisations syndicales etpatronales, les pouvoirs publiques, les associations). La typologie deces parties prenantes donne lieu à de nombreux essais théoriques, et leur mobilisation, à de nombreuses initiatives pratiques $^{6}$. Il demeure en toute hypothèse que leur aptitude à coopérer les unes avec les autres et à faire preuve d'une capacité d'engagement les unes envers les autres est au cœur de l'attention.

\footnotetext{
${ }^{6} \mathrm{~V}$ : Maria Bonnafous-Boucher et Jacob Rendtorff, La Théorie des parties prenantes, Paris, La Découverte, 2014.
} 
Y retrouve-t-on aussi la dimension de l'adossement à la connaissance, de la mise en collaboration concertée de la science et de la pratique (un message très important de la contribution de Jonas)? A dire vrai, c'est moins sûr. Etant née dans un contexte pratique plutôt que dans un environnement scientifique, la RSE n'a cultivé que latéralement la proximité avec les résultats des sciences empiriques, avec la doctrine juridique, avec les développements de l'analyse philosophique ou avec le type de prospective que développement les sciences de l'ingénieur. La « responsabilité sociale des organisations » concerne bien les organisations scientifiques (les universités par exemple) et l' «innovation responsable» donne une place importante aux connaissances scientifiques des ingénieurs ou des chercheurs compétents dans les domaines auxquels on s'intéresse. Toutefois, c'est seulement le mouvement de la RSE en tant que tel qui prend en charge la totalité de la vie de l'entreprise, en lien avec son activité même, davantage que ces rameaux latéraux.

C'est assurément une caractéristique importante de la RSE que l'aspiration à traduire des exigences morales au cœur de la vie des entreprises dans toutes les dimensions de leur activité (la Commission européenne et l'OCDE ont particulièrement mis en valeur cet aspect des choses dans leurs contributions respectives au mouvement général de la « responsabilisation » des entreprises). Or, la RSE comme telle n'a probablement pas réservé une place suffisante à l'insertion des entreprises dans un processus large d'acquisition et de consolidation des connaissances. Dans les faits, les entreprises participent au traitement de l'information et à l'élaboration d'analyses ou de synthèses relevant d'un effort de compréhension, même lorsqu'elles ne se lancent pas dans des activités de recherche "pure ». Les entreprises peuvent contribuer à la manière d'aborder les défis du futur dans la société. Dans différents secteurs - par exemple dans la finance - on peut dessiner les contours, notamment dans la suite des travaux de B. de Bruinn ${ }^{7}$ et de C. Walter ${ }^{8}$ à propos du monde de la finance, d'un problème de «responsabilité cognitive » dans le choix des modèles opératoires qui guident l'action.

\footnotetext{
${ }^{7}$ Boudewijn de Bruin, Ethics and the Global Financial Crisis. Cambridge : Cambridge University Press, 2015.

${ }^{8}$ Christian Walter, Le modèle de marche au hasard en finance, Paris : Economica, 2013. 


\section{Le problème de la responsabilité cognitive}

Jonas a été, dans Le Principe responsabilité et ailleurs, le penseur des rapports entre la théorie et la pratique. Le huitième essai du Phénomène de la vie était consacré aux « usages pratiques de la théorie »?. Tel qu'il se pose aujourd'hui, le problème que l'on a cru pouvoir décrire, ces dernières années, comme le problème de la « responsabilité cognitive » concerne, au-delà de la technologie et des inquiétudes qu'elle éveille, le rapport à la science. Par exemple, en finance, il s'agit du rapport avec les modèles d'évaluation des risques, avec la théorie économique des marchés et avec la théorie politique des rapports entre régulateur et acteurs sociaux. C'est un problème qui concerne souvent le rapport aux recherches en cours de développement. La responsabilité collective face aux «sentiers de l'avenir » que présente la futurologie engage le jugement sur la solidité ou le caractère simplement exploratoire des recherches et des technologies. Ce jugement ne peut pas se résumer à un contrôle des performances ; il engage une évaluation proprement «sociale ${ }^{10}$. Celle-ci ne peut reposer que sur une forme raisonnée de dialogue, sur une base très large.

$\mathrm{Au}$ demeurant, toute la recherche n'a pas vocation à s'appliquer immédiatement, ce qui complique singulièrement les choses pour l'attribution de responsabilité. Le problème se pose avec acuité à cause de l'injonction de faire servir la recherche à l'innovation et au "progrès » (une expression qui est souvent jugée suspecte en philosophie mais qui reste absolument centrale dans la vie institutionnelle concrète). Dans certains cas - par exemple en médecine (saisie dans son rapport à la biologie), en science administrative, en philosophie politique, en éthique, en économie et en finance - les développements des recherches en cours posent en outre un problème relatif à la notion même d'application des conclusions de la recherche. Savoir ce qu'est l'opération consistant à « appliquer» les enseignements de la recherche apparaît de soi beaucoup moins évident que ne l'est, par exemple, la mise en œuvre de la physique des matériaux pour des calculs de résistance des matériaux.

\footnotetext{
${ }^{9}$ Hans Jonas, Le Phénomène de la vie (tr. par Danielle Lories de The Phenomenology of Life. Towards a Philosophical Biology, New York, Harper \& Row, 1966), Paris et Bruxelles, De Boeck Université, 2001.

10 Jean-Jacques Salomon, Le Destin technologique, Paris : Balland, 1992, p. 22. 
C'est aussi dans cette voie de la réflexion sur l'application (technologique ou autre) de la recherche qu'une dimension proprement partenariale de la préparation face à l'avenir est requise. Bien que la RSE n'ait pas mis au centre de ses préoccupations la responsabilité cognitive, plusieurs raisons conduisent à penser qu'un changement est en cours. En particulier, la généralisation de l'approche par les « parties prenantes » se traduit parfois, en pratique, par la participation active des milieux scientifiques. Tel est le cas, en France, avec la plateforme nationale pour la RSE (France-Stratégie), au sein de laquelle le rôle « recherche » (celui des représentants des milieux scientifiques) s'est progressivement affirmé et clarifié, dans un contexte rendu peu consensuel par les incertitudes et les tensions qui entourent le fardeau dont les entreprises doivent accepter de se charger au vu des risques ou enjeux que la recherche et le débat contribuent à préciser. L'approfondissement de la dimension partenariale est une manière d'affronter le problème des biais cognitifs del'expertise et, à bien des égards, elle transforme, en la rendant multilatérale et plus complexe, l'expertise elle-même.

On peut considérer que l'affirmation progressive d'une logique de la vigilance (comme dans la loi française de 2017 sur le «devoir de vigilance» des entreprises) appelle inévitablement un engagement croissant et une responsabilisation des milieux scientifiques et des modélisateurs (dans les sciences de la nature, de la conception, de la société...) pour mieux apprécier les relations causales, les impacts réguliers des activités, le conditionnement des choix par les relations commerciales habituelles, etc. Cet ordre de préoccupations incorpore et généralise pour les entreprises la thématique de la précaution à laquelle l'œuvre de Jonas a donné un grand essor, non sans écho dans les développements institutionnels (d'ordre constitutionnel en France par exemple). De fait, la prise de responsabilité épistémique estl'objet, d'une manière de plus en plus explicite, de recommandations liées à la RSE et qui concernent la recherche de l'objectivité dans l'évaluation des impacts et des progrès des activités (par exemple dans les Recommandations aux entreprises multinationales de l'OCDE).

Dans le message éthique laissé par Jonas, la vigilance côtoie l'appel à la retenue et le refus de la transgression ${ }^{11}$. Dans le monde des organisations qui peuplent notre monde

\footnotetext{
${ }^{11}$ C'est par l'énumération de ces trois termes que J.-J. Salomon (op. cit., p.15) caractérise I' « éthique de la peur » qu'il associe à Jonas.
} 
institutionnel, ces attitudes ne peuvent se décliner en termes simplement psychologiques, ni même dans des termes qui relèvent de l'éclaircissement des conditions de la bonne volonté dans l'engagement moral. Les attitudes qui apparaissent véritablement cruciales sont collectives et se concrétisent par des règles et des procédures mises en œuvre dans les organisations. La question substantielle la plus redoutable, alors, n'est probablement pas celle des « mentalités » et de leur retard perpétuel, ni même celle de l' honnêteté (qui a assurément son importante). C'est plutôt celle de la compatibilité entre les exigences formulées et les pressions sociales qui prennent la forme d'une injonction sociale et institutionnelle maximisatrice - « optimiser en vue de la profitabilité», « rentabiliser » (autant que possible), « exploiter les avantages comparatifs », etc. - ouvrant inévitablement sur les transgressions calculée et le refus de toute retenue. Dans un univers social marqué par la recherche de l'innovation, le statut de la transgression est par ailleurs perpétuellement à préciser ${ }^{12}$.

L'entreprise privée est évidemment au cœur des tensions résultantes, d'une manière toute particulière. Ces tensions sont, très logiquement alors, au cœur du balancement entre une réception de Jonas qui met en évidence sa clairvoyance dans la caractérisation de la responsabilité et une autre réception, moins favorable, qui conduit à poser la question de l' «irresponsabilité » éventuelle d'une conduite prudente mettant les décideurs en porte-à-faux au regard d'attentes sociales qui, souvent, se concrétisent dans des rôles, fonctions, statuts ou professions. Cela peut concerner notamment les attentes contradictoires qui se créent, du fait de la prégnance égale des considérations d'innovation et des considérations de responsabilité sociale, autour de la figure de l'entrepreneneur ${ }^{13}$.

Par ailleurs, les entreprises de régulation donnent une grande place au dialogue institutionnel, à l'explication des actions et des engagements dans le cadre de procédures partiellement codifiées. Dans ces procédures, les acteurs sociaux peuvent

\footnotetext{
${ }^{12}$ On peut s'intéresser à la prévention des transgressions mais aussi, éventuellement, à leur dépassement ou à leur sublimation. V. ainsi, à propos du rôle de l'amour selon Leopold Sedar Senghor : Mireille Alathe Bodo, «Apprendre à être humain : la part de la culture africaine », Diogène, 263-264 (2018), p.44-55; p.51.

${ }^{13}$ Voir ainsi, à propos de Jonas: Emmanuel D'Hombres et Didier Chabanet, «Entre l'homme obligé et I'homme capable : la responsabilité de l'entrepreneur social. Eléments de réflexion phénoménologique», Revue de philosophie économique / Review of Economic Philosophy, 20 (2019), No. 2, p. 105-130; p. 124-125.
} 
mobiliser des connaissances, des théories, des cadres conceptuels explicités, des modèles, etc. De ce fait, une dimension de responsabilité cognitive est engagée. Pour la comprendre, il est aujourd'hui nécessaire de se tourner vers ce qu'il a fallu appelerl' « intelligence artificielle des institutions ${ }^{14}$ (la manière dont les institutions et leurs acteurs se préparent à affronter les situations et à intervenir dans le dialogue). En effet, le raisonnement est à l'œuvre, et il est largement artificiel.

Dans un monde institutionnel, les organisations s'expriment, programment des décisions, exercent des contrôles, organisent et assurent le dialogue sur la base de connaissances empruntées au reste de la société ou élaborées en leur sein. En d'autres termes, la détermination des attitudes à tenir est une affaire raisonnée, qui ne se réduit cependant jamais aux conceptions ou aux volontés d'individus. Le partage du vrai et du faux y joue un rôle, mais aussi la logique des simples acceptations, des choses que l'on accepte de tenir pour vraies ou fausses, des hypothèses de travail ou des repères qui favorisent le travail en commun. Dans un tel univers, la place de la connaissance et de la science est à la fois crucial et mal assurée. Elle n'est pas réservée d'avance : elle se construit plutôt dans des processus et dans le dialogue. Ainsi, les échecs donnent cours à des objections, et l'expérience pratique ne laisse pas intactes les certitudes théoriques. Pour autant, le tribunal de l'expérience laisse toujours subsister de nombreuses incertitudes et ne garantit pas le succès. Par ailleurs, les règles simplement procédurales -à l'exemple d'idéaux délibératifs sur les formes du dialogue satisfaisant, ou de la recherche de « conformité » à des exigences valables par l'entremise de procédures de contrôle - ne sont pas à même de traduire, à elles seules, des manières convaincantes ou éprouvées d'aborder la nouveauté et les difficultés.

En somme, rien ne semble pouvoir épargner le soin de la construction de vertus collectives, dont la nature est intrinsèquement institutionnelle et qui communiquent avec l'innovation, la science et la critique conçues elles aussi comme des complexes collectifs. Dans ces domaines, l'évidence est celle de l'artificialisation croissante des procédures, de la construction des normes et même del'élaboration du dialogue et des attitudes. N'y a-t-il pas ici quelque paradoxe?

\footnotetext{
${ }^{14}$ Cette expression a été introduite dans le cadre de la préparation de la phase II (démarrant en 2019) de la Chaire Ethique et Finance ", Collège d'Etudes Mondiales, Fondation Maison des Sciences de I'Homme, Paris (E. Picavet, C. Walter).
} 
En effet, l'héritage de Jonas en éthique a largement coïncidé avec une sorte de reprise en main de l'éthique par l'ontologie, ou d'adossement des injonctions à la prise de conscience des réalités ${ }^{15}$. Pour autant, prendre au sérieux les préoccupations éthiques auxquelles Jonas a donné une expression peut-être définitives pour le monde technologique affronté aux défis du contrôle social et de la dégradation de l'environnement, cela oblige aujourd'hui à affronter l'artificialisation croissante des décisions humaines et même de la réflexion et du dialogue - en tout cas, pour ce qui concerne la réflexion et le dialogue qui ont un effet pratique à l'échelon le plus décisif, qui est celui des institutions. Prendre au sérieux des préoccupations éthiques substantielles liées à la condition humaine, ce n'est pas seulement un retour à la nature des choses ; c'est aussi une injonction de compréhension et de maîtrise au regard des dispositifs de plus en plus artificiels par lesquels l'homme construit sa pensée, ses conversations, ses attitudes pratiques.

\section{Les défis sociaux et environnementaux et la perspective collective sur les choix individuels problématiques L'importance morale des conditions sociales, technologiques et environnementales de la décision}

La préservation de la figure de l'homme est au cœur d'obligations morales qui conduisent à éviter l'approfondissement des tensions de la volonté. Eviter les dilemmes moraux naissant des conditions de vie et d'interaction sociale, n'est-ce pas une obligation morale en collectivité ? Il se trouve que les développements technologiques ont souvent des conséquences que l'on peut décrire comme la production de tels dilemmes. Ces dilemmes somment l'esprit humain de choisir entre le catastrophique et le pire ; ils imposent des compromissions dans lesquelles l'intégrité humaine risque de se perdre. Certaines situations de choix sont en effet de cette nature et compromettent

\footnotetext{
${ }^{15} \mathrm{~V}$. ainsi, à propos de ce que le recentrement sur la vie apporte à la compréhension concrète du respect de l'homme et du devoir : Eric Pommier, « En quel sens Le principe responsabilitéest-ilun humanisme? ", dans C. Larrère et E. Pommier, L'éthique de la vie chez Hans Jonas, Paris : Publications de la Sorbonne, 2013 ( p. 119-142); p. 120.
} 
la foi dans la destinée humaine autant que l'engagement à perpétuer une image non déformée de l'homme.

Cette préoccupation semble latente chez Jonas, si l'on songe à l'importance qu'il accorde au "comment» de l'existence et au bonum bumanum, à l'intégrité de la représentation de l'homme sur terre comme imago dei, etc. Quoi qu'il en soit de leur arrière-plan théologique, ces notions renvoient au caractère complet, cohérent de la représentation et des visées de l'homme. Décisives pour l'humanisme aujourd'hui, elles peuvent valablement orienter la réflexion éthique même si l'on fait abstraction des schémas culturels ou religieux (dont on peut les juger héritières par ailleurs). Complétude et cohérence doivent fixer l'attention sur les tensions qui traversent la volonté humaine dans son rapport à l'environnement ou aux conditions de vie. Certaines de ces tensions sont suffisantes pour occasionner des choix inexpressifs, voire des choix tragiques. C'est le cas, assez typiquement, dans les situations de « compromis difficile» qui ne permettent pas d'interpréter les choix comme la manifestation de la recherche délibérée d'un bien humain.

\section{Vie et compromis}

Cependant, il y a chez Jonas une tendance à évoquer en termes invariablement plus pressants des enjeux de la vie elle-même comme phénomène physique et biologique (sans exclure pour autant une âpre méditation sur le sacrifice de soi). A vrai dire, ces préoccupations peuvent se rejoindre. Les compromis difficiles peuvent être accentués par l'évolution des systèmes sociaux et par les bouleversements technologiques d'une manière telle qu'ils concernent bien, finalement, la vie et la mort.

Tel est le cas, par exemple, lorsque les routines d'approvisionnement reposent sur une agriculture faisant un large usage de pesticides dangereux. Changer de système ne peut être immédiat et les raisons qui vont dans le sens de la préservation de la santé sont mises en balance, en pratique, avec des raisons qui concernent la sécurité et la suffisance de l'approvisionnement, ainsi que l'accessibilité financière des biens de consommation courante, justement appelés « de première nécessité ». Pour autant, le contexte des échanges et son évolution altèrent véritablement, dans la durée, les 
conditions des choix. Ce qui est "dynamique" et inscrit dans une durée porteuse de changement et d'innovation, ce n'est pas seulement la decision ou le plan que l'on peut programmer d'une manière volontariste, mais aussi les conditions dans lesquelles l'action prend corps.

Le système des échanges et son évolution peuvent donner aux transactions sociales une coloration tragique - car il est bien tragique, par exemple, d'exposer ses enfants à des pesticides nocifs, même si l'on sait que des changements radicaux dans le sens d'une meilleure protection posent des problèmes au système agricole et au commerce des denrées. Jonas plaçait sa confiance dans l'aspiration foncière des hommes à la liberté. Or, il faut bien reconnaître que les conditions tout à la fois matérielles, technologiques et organisationnelles de la vie sociale peuvent ruiner complètement l'aptitude de nos choix volontaires à témoigner des valeurs et de l'intégrité propres à un certain modèle de l'homme (Jonas dirait: une certaine image ou un certain idéal). C'est bien pourquoi il ne saurait y avoir de responsabilité environnementale et technologique pleinement assumée sans responsabilité sociale.

Cet aspect des choses reste à approfondir, certainement dans une certaine continuité par rapport aux réflexions de Jonas. A cet égard, le refus des transgressions arbitraires que l'on associe à juste titre à la pensé de Jonas ne peut se réduire à des listes d'actions “à ne pas faire”. Il engage à rechercher les conditions d'acceptabilité du contexte de l'action. Ce contexte est technologique autant que naturel, mais il est aussi social. Au coeur de cette conjonction, se décident les compromis qui s’imposent aux acteurs dans leurs choix et leurs plans d'action. Si les attentes générales qui se forment au sujet de la préservation de l'existence humaine digne ne doivent pas demeurer vides, elles doivent se concrétiser à ce niveau précisément. Aucune forme de vie humaine n'est véritablement aceptable si elle repose sur des choix dont les conditions sont dégradées au point que leur réforme est impliquée par toute visée substantielle au sujet des destinées de l'homme. En la matière, la glorification abstraite de l'autonomie ou de la liberté entendue comme pure liberté de choix ne saurait suffire, puisque l'action collective donne prise sur l'émergence des termes du choix, autrement dit, des raisons pour lesquelles la promotion de certaines valeurs ou de certains objectifs implique le sacrifice d'autres valeurs ou objectifs (sans nulle garantie a priori d'acceptabilité pour un parti à tenir qui serait accessible). 


\section{La dimension collective de la mauvaise volonté}

Bertrand Saint-Sernin a étudié la possibilité que la volonté humaine puisse devenir authentiquement mauvaise. Le risque serait que nos pouvoirs actifs se réduisent à l'espèce de causalité deficiente en laquelle consiste la mauvaise volonté dans une tradition venue notamment d'Augustin d'Hippone. Si l'évocation de ce danger s'est enracinée, chez B. Saint-Sernin, dans la réflexion sur le destin des technosciences et de leur impact sur la prise de decision ${ }^{16}$, elle a également partieliée avec l'étude des caractéristiques de la vie institutionnelle. Elleparticipe, comme chez Jonas, de la prise de conscience du poids de routines qui penchent davantage du côté de la pesanteur que du côté de la grâce (pour parler comme Simone Weil) et qui peuvent être des rouages de l"'apocalypse rampante" redoutée par Jonas. Au-delà donc de la présupposition « métaphysique » admise par Jonas - celle de la valeur intrinsèque ajoutée au monde par la présence de notre volonté - il faudrait être attentif, si l'on suit cette voie, au mal et au non-sens. Cela peut prendre un tour interrogatif: est-ce que notre volonté dans le monde et la responsabilité qui l'accompagne font signe vers le bien?

Cette question prend place dans la philosophie contemporaine notamment dans la perspective d'une interrogation existentielle face aux destinées que semblent nous imposer les sciences et les techniques, et face aux transformations des conditions environnementales, technologiques et organisationnelles de la décision.

Dans ce tableau, il faut inclure la vie propre aux institutions. La décision n'est pas seulement l'occasion d'exprimer des valeurs ; à travers les conditions de sa formation et de son inscription dans le monde, elle est aussi un marqueur de la possibilité même d'exprimer des valeurs dans la vie et dans l'action. Or, cette possibilité est très largement subordonnée, dans le monde tel qu'il est, aux procédures, aux initiatives et aux prises de parole que consacrent ou rejettent les institutions qui structurent la vie sociale et les organisations particulières.

Disons aussi que la question du rapport entre le bien et la volonté est ce qui vient limiter a priori la pertinence de toute velléité de transformation de l'exigence

\footnotetext{
${ }^{16}$ B. Saint-Sernin, Parcours de l'ombre. Les trois indécidable, Bruxelles : Editions des Archives contemporaines, 1994 ; La raison au vingtième siècle, Paris : Seuil, 1995.
} 
morale en une simple éthique de l'accompagnement des transformations. Le traitement de cette question ne peut s'épanouir que dans une philosophie sociale capable d'inspirer occasionnellement la résistance aux transformations, dans la mesure exacte où elle sonde les conditions du développement d'un exercice serein, rationnel et expressif de la responsabilité. Si l'on néglige cette perspective, le risque l'appauvrissement principiel de la volonté dans l'action, voisinant finalement avec l'abandon de la liberté.

Nous ne devrions pas alors en rester à l'examen de la contribution de la société aux bases matérielles, proprement biologiques au sens courant, de la vie partagée. La démarche devrait concerner en droit aussi la préservation ou la reconstitution de cadres sociaux corrects de l'action, fournissant des termes des choix, de l'interprétation et de la responsabilité qui soient pleinement significatifs pour l'être humain. C'est une préoccupation importante de la philosophie contemporaine, qui s'intéresse de près à des thèmes tels que la dépendance et l'interdépendance, les limites de l'exercice des facultés mobilisées dans les diverses conceptions de l'autonomie et du libre choix, ou encore les conditions collectives et institutionnelles de la « liberté réelle ».

Dans ces travaux, il est souvent question de l'ampleur ou de la restriction des choix possibles (comme dans les approches économiques de la valeur des «ensembles de choix»), de l'égalité ou de l'équité dans la disponibilité de moyens d'action que l'on souhaite aussi grands que possible (comme dans les recherches sur l'égalité et les inégalités sociales), du renforcement de capacités d'action jugées souhaitables (comme dans le thème de l'empowerment dans le monde anglophone), ou encore de la valeur équitable de l'usage des libertés ou droits qui sont attribués ou garantis (dans la continuité des analyses de John Rawls). Un point commun fréquent des explorations de ces thématiques - ces dernières étant d'ailleurs importantes en elle-même - est de laisser de côté la question du caractère souhaitable ou non de l'accroissement des facultés d'action qui sous-tendent la décision.

Cela intervient le plus souvent par méthode (parce que l'on s'intéresse sélectivement à des questions de justice ou d'égalité), et parfois par conviction « libérale », parce que l'on estime que le maximum de disponibilité et d'usage de facultés de choix est en soi une bonne chose au regard de la vie des êtres autonomes que les êtres 
humains sont appelés à être ${ }^{17}$. Toutefois, une perspective collective sur la décision individuelle et sur les interactions en sous-groupes est nécessaire à bien des égards. Elle est nécessaire à l'échelle macroscopique qui a prévalu dans la réception du Principe responsabilité de Jonas: pour la survie de l'humanité, pour la continuité de la vie, pour la préservation des écosystèmes. Elle est aujourd'hui capitale pour RSE, dont la dimension morale tient justement dans la reconnaissance volontaire de limites de l'action et de l'impact de l'activité sur autrui.

Dans une perspective plus proche de l'échelon individuel, on peut aussi dire que la perspective collective, nourrie par le type de futurologie que Jonas a appelé de ses vœux et dont il a fourni l'esquisse, est importante pour la possibilité maintenue de l'action et de la décision dans leurs dimensions pleinement humaines. Ne devons- nous pas veiller à parer le risque de leur enfermement contextuel dans des situations absurdes, dans des dilemmes insolubles? Ou dans des compromissions qui sont simultanément inévitables (dans des contextes émergents) et fatales à l'intégrité des personnes ? Ces questions doivent certainement accompagner la réflexion sur l'environnement et sur les techniques.

\section{La préservation des cadres de l'action, à l'horizon de la pensé de Jonas et des défis contemporains}

Le vingtième siècle n'a certes pas été propice à l'épanouissement d'une vision hégélienne de l'éthique, selon laquelle l'accomplissement plein et entier de celle-ci passerait par la réalisation de l'esprit objectif et pourrait donner aux hommes et à l'action humaine les cadres qui leur conviennent. Bien plutôt, le vingtième siècle a conduit les acteurs de ses moments tragiques à reconnaître la morale toute pure dans la révolte de la seule conscience, dans la défiance à l'endroit des cadres sociaux corrompus, dans le mépris des compromissions faussement prudentes et dans la confiance dans la certitude subjective de bien agir. Simultanément, les formes plus faibles d' « éthique » politique, professionnelle ou institutionnalisée apparaissent

\footnotetext{
${ }^{17}$ V. en ce sens: Catherine Audard, Qu'est-ce que le libéralisme? Paris, Gallimard, 2009. 
souvent comme une sorte de gestion des maux, qui peut voisiner avec ce qui est perçu comme franchement mauvais globalement.

Cependant, la reconstruction de la société humaine après les effroyables tragédies du vingtième siècle s’inscrit dans la durée et elle suppose bien à ce titre la reconquête et l'aménagement concerté des institutions. Cette tâche inachevée, et qui connaît des retours en arrière, demande d'aller au-delà de la moralité du pur engagement personnel. Elle rend nécessaire de s'intéresser aux cadres sociaux de l'action et de maintenir un rapport individuel et collectif critique à ces cadres. Dans cette perspective, si l'on est préoccupé par la préservation de l'exercice de la volonté libre, l'attention doit certainement se porter sur notre responsabilité dans l'évitement d'un déchirement au centre de la raison pratique.

Celle-ci peut en effet être tiraillée, à notre corps défendant, dans des dilemmes moraux que les évolutions technologiques et socio-économiques contribuent à approfondir, ou à créer. Ainsi, l'exacerbation des tensions occasionnées par la rareté de biens humains nécessaires ou importants est une source de situations de choix dans lesquelles on perd de vue la recherche du bien dans et par l'action humaine. L'enfermement de l'individu dans des groupes communautaires, ayant pour résultat que de nombreux choix par ailleurs légitimes apparaissent a priori porteurs du reniement d'une identité, est aussi problématique dans la même perspective. Il est en effet de nature à inscrire dans les contextes de l'action des dilemmes pratiques qui compromettent le libre exercice de la rationalité pratique et du jugement, en divisant artificiellement l'agent lui-même et en menaçant la figure - porteuse de sens et de valeur - d'un jugement tout à la fois librement formé et capable d'inspirer l'action.

Face à de tels défis, la prise de responsabilité des entreprises que favorise le mouvement de la RSE est importante parce qu'elle restitue, au cœur des valeurs de l'action proprement institutionnelle, un référentiel axiologique explicite ${ }^{18} 16$. Elle prétend bien structurer l'action par la recherche d'un bien humain. Bien entendu, le rapport au bien est ici assez différent de la visée purement individuelle du bien et cela appelle une prise en compte des spécificités des endossements, acceptations et

\footnotetext{
${ }^{18}$ Cette caractéristique de la RSE conditionne son inscription dans des dynamiques de progrès. V. à ce propos : E. Picavet, «Entre normes et stratégie : quelques apports de la Responsabilité Sociale des Entreprises », Rue Descartes 2017 / 1 ( N 91), p. $62-75$.
} 
communications institutionnelles. Le partage des rôles ou des responsabilités institutionnelles prend aussi de l'importance dans le contexte de la RSE et il ne peut se réduire à l'idée simpliste d'une « prise de relais » entre l'Etat et les entreprises.

Si flous que soient ses contours (comme en témoignent les discussions juridiques européennes autour de la concordance ou non entre et l'undertaking anglais et l'entreprise), l'entreprise est une institution dont les opérations ne peuvent se comprendre que dans l'interaction avec d'autres institutions. C'est seulement dans cette interaction que la prise de responsabilité a un sens. Or, cette interaction illustre tout à la fois le potentiel de l'engagement des entreprises et son insuffisance, si les pouvoirs publics n'assument pas un rôle actif dans la promotion de conditions correctes des interactions économiques et des choix sociaux, technologiques et environnementaux des acteurs privés.

\section{Conclusion}

L'évitement ou l'affaiblissement des tensions que nous avons évoquées à propos de l'action humaine a été notamment l'œuvre historique, insuffisamment connue et valorisée, de la formation de l'Etat social. Plus récemment, l'essor des préoccupations écologiques et la quête d'un développement durable ont donné de l'élan à la quête d'une reconnaissance objective, dans les cadres sociaux, des efforts moraux de mise en œuvre de principes dont l'importance est éprouvée pour l'action humaine responsable.

En sens inverse, la manière politique de favoriser une concurrence économique indifférente aux conditions d'emploi, aux normes environnementales et aux systèmes sociaux est de nature à enfermer les personnes dans une logique de réponse aux contraintes. De même, l'approfondissement toujours croissant des modèles gestionnaires reposant sur la "responsabilisation" individuelle au regard d'objectifs donnés d'avance est de nature à paralyser le sens critique, le libre dialogue et l'ouverture à la connaissance, tout autant que la volontée réfléchie d'inscrire sa propre action dans la concertation, dans l'action collective, dans l'effort partagé pour faire 
évoluer dans une direction favorable (et tout d'abord acceptable) les conditions de la vie institutionnelle.

Dans un contexte social et environnemental appauvri de l'action, la liberté résiduelle se réduit trop souvent au sacrifice d'une exigence importante au profit d'une autre exigence importante (choisir par exemple des dépenses de santé au détriment de dépenses d'éducation dans des systèmes sociaux où l'éducation et la santé occasionnent des frais importants et non collectivisés). On a peine, alors, à participer de son plein gré à ce mouvement général d'amélioration de l'action des uns et des autres, dans lequel Bergson avait su reconnaitre la vraie marque du progrès moral rendu possible par les initiatives d'individus novateurs ${ }^{19}$.

De ce point de vue, l'insistance de Jonas sur les conditions collectives de la préparation à l'avenir dans un souci qui est simultanément le souci du présent doit certainement conduire à rapprocher l'éthique de l'analyse institutionnelle des capacités d'action sociale et environnementale. Elle oblige également à affronter les nombreux problèmes qu'enveloppe l'artificialisation croissante des dispositions typiques de la réflexion, de la coordination et de la coopération, du dialogue et de la délibération, dans les cadres institutionnels de l'action. Certains de ces problèmes, en effet, sont devenus véritablement constitutifs de la possibilité même d'agir d'une manière qui soit expressive au regard d'attentes enracinées dans l'éthique.

\section{Bibliographie}

ALATHE BODO, M. Apprendre à être humain : la part de la culture africaine. Diogène, 263264 (2018), p. 44-55; p. 51.

AUDARD, C. Qu'est-ce que le libéralisme? Paris, Gallimard, 2009.

BONNAFOUS-BOUCHER, M., RENDTORFF, J. La Théorie des parties prenantes. Paris: La Découverte, 2014.

BERGSON, H. Les Deux sources de la morale et de la religión. Paris: Félix Alcan, 1932.

De BRUIN, B. Ethics and the Global Financial Crisis. Cambridge : Cambridge University Press, 2015.

\footnotetext{
${ }^{19}$ Bergson, H., Les Deux sources de la morale et de la religion, Paris: Félix Alcan, 1932. 
D'HOMBRES, E., CHABANET, D. Entre l'homme obligé et l'homme capable : la responsabilité de l'entrepreneur social. Eléments de réflexion phénoménologique. Revue de philosophieéconomique / Review ofEconomicPhilosophy, 20 (2019),No.2,p.105-130.

DUPUY, J.-P. Pour un catastrophisme éclairé. Paris: Seuil, 2002.

JONAS, H. Le Phénomène de la vie (tr. par Danielle Lories de The Phenomenon of Life. Towards a Philosophical Biology, New York, Harper \& Row, 1966), Paris et Bruxelles, De Boeck Université, 2001.

JONAS, H. Le principe responsabilité (tr. fr. Jean Greisch de Das Prinzip Verantwortung, Francfort, Insel Verlag, 1979). Paris : Les Editions du Cerf, 1990.

JONAS, H. Pour une éthique du futur (tr. par S. Cornille et Ph. Ivernel de Philosophie, Rückschau und Vorschau am Ende des Jabrbunderts [Frankfurt, Suhrkamp 1993] et partiellement de Philosophische Untersuchungen und metaphysische Vermutungen [Frankfurt am Main \& Leipzig, Insel Verlag, 1992]. Paris : Payot et Rivages, 1998.

PICAVET, E. Entre normes et stratégie : quelques apports de la Responsabilité Sociale des Entreprises. Rue Descartes 2017 / 1 (N 91), p.62- 75.

POMMIER, E. En quel sens Le principe responsabilité est-il un humanisme ? In : C. Larrère, E. Pommier, L'éthique de la vie chez. Hans Jonas, Paris : Publications de la Sorbonne, 2013, p. 119-142.

SAINT-SERNIN, B. Parcours de l'ombre. Les trois indécidables. Bruxelles : Editions des Archives contemporaines, 1994.

SAINT-SERNIN, B. La raison au vingtième siècle. Paris: Seuil, 1995.

SALOMON, J.- J. Le Destin technologique, Paris : Balland, 1992.

SERIS, J.-P. La Technique. Paris: Presses Universitaires de France, 2014.

WALTER, C. Le modèle de marche au hasard en finance, Paris : Economica, 2013. 